\title{
Paraphrases as Foreign Languages in Multilingual Neural Machine Translation
}

\author{
Zhong Zhou \\ Carnegie Mellon University \\ zhongzhoud cmu.edu
}

\author{
Matthias Sperber \\ Karlsruhe Institute of Technology \\ matthias.sperber@kit.edu
}

\author{
Alex Waibel \\ Carnegie Mellon University \\ Karlsruhe Institute of Technology \\ alex@waibel.com
}

\begin{abstract}
Paraphrases, the rewordings of the same semantic meaning, are useful for improving generalization and translation. However, prior works only explore paraphrases at the word or phrase level, not at the sentence or corpus level. Unlike previous works that only explore paraphrases at the word or phrase level, we use different translations of the whole training data that are consistent in structure as paraphrases at the corpus level. We train on parallel paraphrases in multiple languages from various sources. We treat paraphrases as foreign languages, tag source sentences with paraphrase labels, and train on parallel paraphrases in the style of multilingual Neural Machine Translation (NMT). Our multi-paraphrase NMT that trains only on two languages outperforms the multilingual baselines. Adding paraphrases improves the rare word translation and increases entropy and diversity in lexical choice. Adding the source paraphrases boosts performance better than adding the target ones. Combining both the source and the target paraphrases lifts performance further; combining paraphrases with multilingual data helps but has mixed performance. We achieve a BLEU score of 57.2 for French-to-English translation using 24 corpus-level paraphrases of the Bible, which outperforms the multilingual baselines and is +34.7 above the single-source singletarget NMT baseline.
\end{abstract}

\section{Introduction}

Paraphrases, rewordings of texts with preserved semantics, are often used to improve generalization and the sparsity issue in translation (CallisonBurch et al., 2006; Fader et al., 2013; Ganitkevitch et al., 2013; Narayan et al., 2017; Sekizawa et al., 2017). Unlike previous works that use paraphrases at the word/phrase level, we research on different translations of the whole corpus that are consistent in structure as paraphrases at the corpus level;

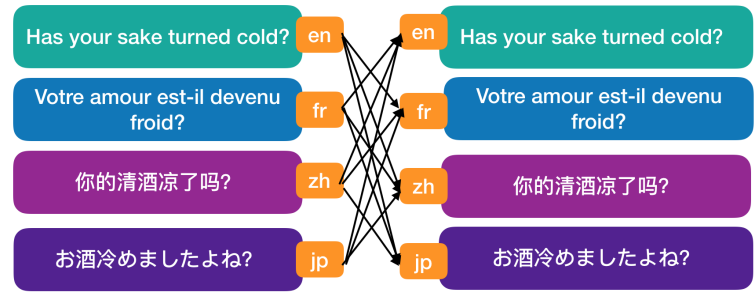

(a) multilingual NMT

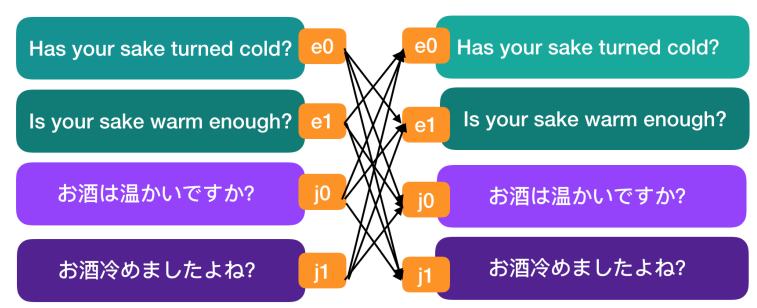

(b) multi-paraphrase NMT

Figure 1: Translation Paths in (a) multilingual NMT (b) multi-paraphrase NMT. Both form almost a complete bipartite graph.

we refer to paraphrases as the different translation versions of the same corpus. We train paraphrases in the style of multilingual NMT (Johnson et al., 2017; Ha et al., 2016) . Implicit parameter sharing enables multilingual NMT to learn across languages and achieve better generalization (Johnson et al., 2017). Training on closely related languages are shown to improve translation (Zhou et al., 2018). We view paraphrases as an extreme case of closely related languages and view multilingual data as paraphrases in different languages. Paraphrases can differ randomly or systematically as each carries the translator's unique style.

We treat paraphrases as foreign languages, and train a unified NMT model on paraphrase-labeled data with a shared attention in the style of multilingual NMT. Similar to multilingual NMT's objective of translating from any of the $N$ input languages to any of the $M$ output languages (Firat et al., 2016), multi-paraphrase NMT aims to translate from any of the $N$ input paraphrases to any 
of the $M$ output paraphrases in Figure 1. In Figure 1, we see different expressions of a host showing courtesy to a guest to ask whether sake (a type of alcohol drink that is normally served warm in Asia) needs to be warmed. In Table 6, we show a few examples of parallel paraphrasing data in the Bible corpus. Different translators' styles give rise to rich parallel paraphrasing data, covering wide range of domains. In Table 7, we also show some paraphrasing examples from the modern poetry dataset, which we are considering for future research.

Indeed, we go beyond the traditional NMT learning of one-to-one mapping between the source and the target text; instead, we exploit the many-to-many mappings between the source and target text through training on paraphrases that are consistent to each other at the corpus level. Our method achieves high translation performance and gives interesting findings. The differences between our work and the prior works are mainly the following.

Unlike previous works that use paraphrases at the word or phrase level, we use paraphrases at the entire corpus level to improve translation performance. We use different translations of the whole training data consistent in structure as paraphrases of the full training data. Unlike most of the multilingual NMT works that uses data from multiple languages, we use paraphrases as foreign languages in a single-source single-target NMT system training only on data from the source and the target languages.

Our main findings in harnessing paraphrases in NMT are the following.

1. Our multi-paraphrase NMT results show significant improvements in BLEU scores over all baselines.

2. Our paraphrase-exploiting NMT uses only two languages, the source and the target languages, and achieves higher BLEUs than the multi-source and multi-target NMT that incorporates more languages.

3. We find that adding the source paraphrases helps better than adding the target paraphrases.

4. We find that adding paraphrases at both the source and the target sides is better than adding at either side.
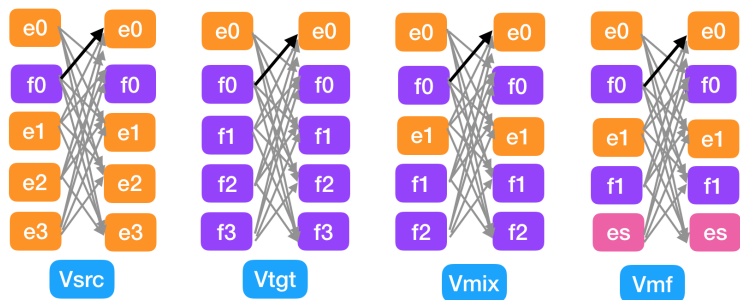

Figure 2: Examples of different ways of adding 5 paraphrases. $e[? n]$ and $f[? n]$ refers to different English and French paraphrases, es refers to the Spanish (an example member of Romance family) data. We always evaluate the translation path from $\mathrm{f} 0$ to $\mathrm{e} 0$.

5. We also find that adding paraphrases with additional multilingual data yields mixed performance; its performance is better than training on language families alone, but is worse than training on both the source and target paraphrases without language families.

6. Adding paraphrases improves the sparsity issue of rare word translation and diversity in lexical choice.

In this paper, we begin with introduction and related work in Section 1 and 2. We introduce our models in Section 3. Finally, we present our results in Section 4 and conclude in Section 5.

\section{Related Work}

\subsection{Paraphrasing}

Many works generate and harness paraphrases (Barzilay and McKeown, 2001; Pang et al., 2003; Callison-Burch et al., 2005; Mallinson et al., 2017; Ganitkevitch et al., 2013; Brad and Rebedea, 2017; Quirk et al., 2004; Madnani et al., 2012; Suzuki et al., 2017; Hasan et al., 2016). Some are on question and answer (Fader et al., 2013; Dong et al., 2017), evaluation of translation (Zhou et al., 2006) and more recently NMT (Narayan et al., 2017; Sekizawa et al., 2017). Past research includes paraphrasing unknown words/phrases/subsentences (Callison-Burch et al., 2006; Narayan et al., 2017; Sekizawa et al., 2017; Fadaee et al., 2017). These approaches are similar in transforming the difficult sparsity problem of rare words prediction and long sentence translation into a simpler problem with known words and short sentence translation. It is worthwhile to contrast paraphrasing that diversifies data, with knowledge distillation that benefits from making data more consistent (Gu et al., 2017).

Our work is different in that we exploit paraphrases at the corpus level, rather than at the word 


\begin{tabular}{|l|l|l|l|l|}
\hline Data & 1 & 6 & 11 & 13 \\
\hline \hline VsrC & 22.5 & 41.4 & 48.9 & 48.8 \\
\hline Vtgt & 22.5 & 40.5 & 47.0 & 47.4 \\
\hline
\end{tabular}

Table 1: Comparison of adding source paraphrases and adding target paraphrases. All acronyms including data are explained in Section 4.3.

\begin{tabular}{|l|l|l|l|l|l|l|}
\hline data & 1 & 6 & 11 & 16 & 22 & 24 \\
\hline \hline WMT & 22.5 & 30.8 & 29.8 & 30.8 & 29.3 & - \\
\hline Family & 22.5 & 39.3 & 45.4 & 49.2 & 46.6 & - \\
\hline Vmix & 22.5 & 44.8 & 50.8 & 53.3 & 55.4 & 57.2 \\
\hline Vmf & - & - & 49.3 & - & - & - \\
\hline
\end{tabular}

Table 2: Comparison of adding a mix of the source paraphrases and the target paraphrases against the baselines. All acronyms including data are explained in Section 4.3.

or phrase level.

\subsection{Multilingual Attentional NMT}

Machine polyglotism which trains machines to translate any of the $N$ input languages to any of the $M$ output languages from many languages to many languages, many languages is a new paradigm in multilingual NMT (Firat et al., 2016; Zoph and Knight, 2016; Dong et al., 2015; Gillick et al., 2016; Al-Rfou et al., 2013; Tsvetkov et al., 2016). The objective is to translate from any of the $N$ input languages to any of the $M$ output languages (Firat et al., 2016).

Many multilingual NMT systems involve multiple encoders and decoders (Ha et al., 2016), and it is hard to combine attention for quadratic language pairs bypassing quadratic attention mechanisms (Firat et al., 2016). An interesting work is training a universal model with a shared attention mechanism with the source and target language labels and Byte-Pair Encoding (BPE) (Johnson et al., 2017; Ha et al., 2016). This method is elegant in its simplicity and its advancement in low-resource language translation and zero-shot translation using pivot-based translation mechanism (Johnson et al., 2017; Firat et al., 2016).

Unlike previous works, our parallelism is across paraphrases, not across languages. In other words, we achieve higher translation performance in the single-source single-target paraphrase-exploiting NMT than that of the multilingual NMT.

\section{Models}

We have four baseline models. Two are singlesource single-target attentional NMT models, the other two are multilingual NMT models with a shared attention (Johnson et al., 2017; Ha et al., 2016). In Figure 1, we show an example of multilingual attentional NMT. Translating from all 4 languages to each other, we have 12 translation paths. For each translation path, we label the source sentence with the source and target language tags. Translating from “你的清 酒凉了吗?" to “Has your sake turned cold?", we label the source sentence with _-opt_src_zh _-opt_tgt_en. More details are in Section 4.

In multi-paraphrase model, all source sentences are labeled with the paraphrase tags. For example, in French-to-English translation, a source sentence may be tagged with _-opt_src_f1 _-opt_tgt_e 0 , denoting that it is translating from version " 11 " of French data to version "e0" of English data. In Figure 1, we show 2 Japanese and 2 English paraphrases. Translating from all 4 paraphrases to each other $(N=M=4)$, we have 12 translation paths as $N \times(N-1)=12$. For each translation path, we label the source sentence with the source and target paraphrase tags. For the translation path from “お酒冷めましたよね?” to "Has your sake turned cold?", we label the source sentence with _-opt_src_j1 _opt_tgt_e 0 in Figure 1. Paraphrases of the same translation path carry the same labels. Our paraphrasing data is at the corpus level, and we train a unified NMT model with a shared attention. Unlike the paraphrasing sentences in Figure 1, We show this example with only one sentence, it is similar when the training data contains many sentences. All sentences in the same paraphrase path share the same labels.

\section{Experiments and Results}

\subsection{Data}

Our main data is the French-to-English Bible corpus (Mayer and Cysouw, 2014), containing 12 versions of the English Bible and 12 versions of the French Bible ${ }^{1}$. We translate from French to English. Since these 24 translation versions are consistent in structure, we refer to them as paraphrases at corpus level. In our paper, each paraphrase refers to each translation version of whole Bible corpus. To understand our setup, if we use all 12 French paraphrases and all 12 English paraphrases so there are 24 paraphrases in total, i.e., $N=M=24$, we have 552 translation paths be-

\footnotetext{
${ }^{1}$ We considered the open subtitles with different scripts of the same movie in the same language; they covers many topics, but they are noisy and only differ in interjections. We also considered the poetry dataset where a poem like "If" by Rudyard Kipling is translated many times, by various people into the same language, but the data is small.
} 


\begin{tabular}{|c|c|c|}
\hline Source Sentence & Machine Translation & Correct Target Translation \\
\hline $\begin{array}{l}\text { Comme de l'eau fraîche pour une per- } \\
\text { sonne fatigué, Ainsi est une bonne } \\
\text { nouvelle venant d'une terre lointaine. }\end{array}$ & $\begin{array}{l}\text { As cold waters to a thirsty soul, so is } \\
\text { good news from a distant land. }\end{array}$ & $\begin{array}{l}\text { Like cold waters to a weary soul, so is } \\
\text { a good report from a far country. }\end{array}$ \\
\hline $\begin{array}{l}\text { Lorsque tu seras invité par quelqu'un à } \\
\text { des noces, ne te mets pas à la première } \\
\text { place, de peur qu'il n'y ait parmi les } \\
\text { invités une personne plus considérable } \\
\text { que toi, }\end{array}$ & $\begin{array}{l}\text { When you are invited to one to the } \\
\text { wedding, do not be to the first place, } \\
\text { lest any one be called greater than you. }\end{array}$ & $\begin{array}{l}\text { When you are invited by anyone to } \\
\text { wedding feasts, do not recline at the } \\
\text { chief seat lest one more honorable than } \\
\text { you be invited by him, }\end{array}$ \\
\hline $\begin{array}{l}\text { Car chaque arbre se connaît à son fruit. } \\
\text { On ne cueille pas des figues sur des } \\
\text { épines, et l'on ne vendange pas des } \\
\text { raisins sur des ronces. }\end{array}$ & $\begin{array}{l}\text { For each tree is known by its own fruit. } \\
\text { For from thorns they do not gather figs, } \\
\text { nor do they gather grapes from a bram- } \\
\text { ble bush. }\end{array}$ & $\begin{array}{l}\text { For each tree is known from its own } \\
\text { fruit. For they do not gather figs from } \\
\text { thorns, nor do they gather grapes from } \\
\text { a bramble bush. }\end{array}$ \\
\hline $\begin{array}{l}\text { Vous tous qui avez soif, venez aux } \\
\text { eaux, Même celui qui n'a pas d'argent! } \\
\text { Venez, achetez et mangez, Venez, } \\
\text { achetez du vin et du lait, sans argent, } \\
\text { sans rien payer! }\end{array}$ & $\begin{array}{l}\text { Come, all you thirsty ones, come to the } \\
\text { waters; come, buy and eat. Come, buy } \\
\text { for wine, and for nothing, for without } \\
\text { money. }\end{array}$ & $\begin{array}{l}\text { Ho, everyone who thirsts, come to the } \\
\text { water; and he who has no silver, come } \\
\text { buy grain and eat. Yes, come buy } \\
\text { grain, wine and milk without silver and } \\
\text { with no price. }\end{array}$ \\
\hline $\begin{array}{l}\text { Oui, vous sortirez avec joie, Et vous } \\
\text { serez conduits en paix ; Les montagnes } \\
\text { et les collines éclateront d'allégresse } \\
\text { devant vous, Et tous les arbres de la } \\
\text { campagne battront des mains. }\end{array}$ & $\begin{array}{l}\text { When you go out with joy, you shall } \\
\text { go in peace ; the mountains shall re- } \\
\text { joice before you, and the trees of the } \\
\text { field shall strike all the trees of the field } \\
\text {. }\end{array}$ & $\begin{array}{l}\text { For you shall go out with joy and be led } \\
\text { out with peace. The mountains and the } \\
\text { hills shall break out into song before } \\
\text { you, and all the trees of the field shall } \\
\text { clap the palm. }\end{array}$ \\
\hline
\end{tabular}

Table 3: Examples of French-to-English translation trained using 12 French paraphrases and 12 English paraphrases.

cause $N \times(N-1)=552$. The original corpus contains missing or extra verses for different paraphrases; we clean and align 24 paraphrases of the Bible corpus and randomly sample the training, validation and test sets according to the 0.75 , $0.15,0.10$ ratio. Our training set contains only $23 \mathrm{~K}$ verses, but is massively parallel across paraphrases.

For all experiments, we choose a specific English corpus as $e 0$ and a specific French corpus as $\mathrm{f} 0$ which we evaluate across all experiments to ensure consistency in comparison, and we evaluate all translation performance from $\mathrm{f} 0$ to $e 0$.

\subsection{Training Parameters}

In all our experiments, we use a minibatch size of 64, dropout rate of 0.3, 4 RNN layers of size 1000, a word vector size of 600 , number of epochs of 13 , a learning rate of 0.8 that decays at the rate of 0.7 if the validation score is not improving or it is past epoch 9 across all LSTM-based experiments. Byte-Pair Encoding (BPE) is used at preprocessing stage (Ha et al., 2016). Our code is built on OpenNMT (Klein et al., 2017) and we evaluate our models using BLEU scores (Papineni et al., 2002), entropy (Shannon, 1951), F-measure and qualitative evaluation.

\subsection{Baselines}

We introduce a few acronyms for our four baselines to describe the experiments in Table 1, Table 2 and Figure 3. Firstly, we have two single-source single-target attentional NMT mod- els, Single and WMT. Single trains on $\mathrm{f} 0$ and $e 0$ and gives a BLEU of 22.5, the starting point for all curves in Figure 3. WMT adds the out-domain WMT'14 French-to-English data on top of $\mathrm{f} 0$ and $e 0$; it serves as a weak baseline that helps us to evaluate all experiments' performance discounting the effect of increasing data.

Moreover, we have two multilingual baselines ${ }^{2}$ built on multilingual attentional NMT, Family and Span (Zhou et al., 2018). Family refers to the multilingual baseline by adding one language family at a time, where on top of the French corpus $f 0$ and the English corpus e0, we add up to 20 other European languages. Span refers to the multilingual baseline by adding one span at a time, where a span is a set of languages that contains at least one language from all the families in the data; in other words, span is a sparse representation of all the families. Both Family and Span trains on the Bible in 22 Europeans languages trained using multilingual NMT. Since Span is always suboptimal to Family in our results, we only show numerical results for Family in Table 1 and 2, and we plot both Family and Span in Figure 3. The two multilingual baselines are strong baselines while the fWMT baseline is a weak baseline that helps us to evaluate all experiments' performance discounting the effect of increasing data. All baseline results are taken from

\footnotetext{
${ }^{2}$ For multilingual baselines, we use the additional Bible corpus in 22 European languages that are cleaned and aligned to each other.
} 


\begin{tabular}{|l|l|l|l|l|l|}
\hline data & 6 & 11 & 16 & 22 & 24 \\
\hline \hline Entropy & 5.6569 & 5.6973 & 5.6980 & 5.7341 & 5.7130 \\
\hline Bootstr. & 5.6564 & 5.6967 & 5.6975 & 5.7336 & 5.7125 \\
$95 \%$ CI & 5.6574 & 5.6979 & 5.6986 & 5.7346 & 5.7135 \\
\hline WMT & - & 5.7412 & 5.5746 & 5.6351 & - \\
\hline
\end{tabular}

Table 4: Entropy increases with the number of paraphrase corpora in Vmix. The 95\% confidence interval is calculated via bootstrap resampling with replacement.

\begin{tabular}{|l|l|l|l|l|l|}
\hline data & 6 & 11 & 16 & 22 & 24 \\
\hline \hline F1(freq1) & 0.43 & 0.54 & 0.57 & 0.58 & 0.62 \\
\hline WMT & - & 0.00 & 0.01 & 0.01 & - \\
\hline
\end{tabular}

Table 5: F1 score of frequency 1 bucket increases with the number of paraphrase corpora in $\operatorname{Vmix}$, showing training on paraphrases improves the sparsity at tail and the rare word problem.

a research work which uses the grid of $(1,6,11$, $16,22)$ for the number of languages or equivalent number of unique sentences and we follow the same in Figure 3 (Zhou et al., 2018). All experiments for each grid point carry the same number of unique sentences.

Furthermore, VSrC refers to adding more source (English) paraphrases, and Vtgt refers to adding more target (French) paraphrases. $V m i x$ refers to adding both the source and the target paraphrases. $V m f$ refers to combining $V m i x$ with additional multilingual data; note that only $\mathrm{Vmf}$, Family and Span use languages other than French and English, all other experiments use only English and French. For the $\mathrm{x}$-axis, data refers to the number of paraphrase corpora for $\operatorname{VsrC}$, Vtgt, Vmix; data refers to the number of languages for Family; data refers to and the equivalent number of unique training sentences compared to other training curves for $W M T$ and $V m f$.

\subsection{Results}

Training on paraphrases gives better performance than all baselines: The translation performance of training on 22 paraphrases, i.e., 11 English paraphrases and 11 French paraphrases, achieves a BLEU score of 55.4, which is +32.9 above the Single baseline, +8.8 above the Fami Iy baseline, and +26.1 above the WMT baseline. Note that the Family baseline uses the grid of $(1,6,11,16,22)$ for number of languages, we continue to use this grid for our results on number of paraphrases, which explains why we pick 22 as an example here. The highest BLEU 57.2 is achieved when we train on 24 paraphrases, i.e., 12 English paraphrases and 12 French paraphrases.

Adding the source paraphrases boosts translation performance more than adding the tar-

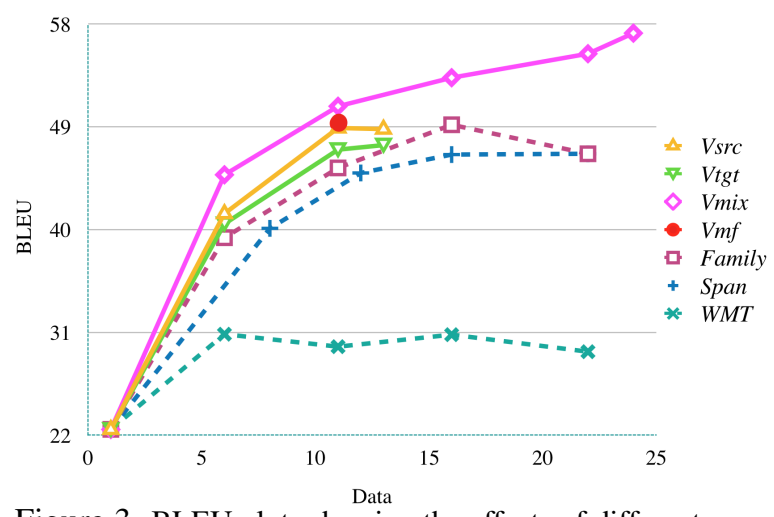

Figure 3: BLEU plots showing the effects of different ways of adding training data in French-to-English Translation. All acronyms including data are explained in Section 4.3.

get paraphrases: The translation performance of adding the source paraphrases is higher than that of adding the target paraphrases. Adding the source paraphrases diversifies the data, exposes the model to more rare words, and enables better generalization. Take the experiments training on 13 paraphrases for example, training on the source (i.e., 12 French paraphrases and the English paraphrase e0) gives a BLEU score of 48.8, which has a gain of +1.4 over 47.4 , the BLEU score of training on the target (i.e., 12 English paraphrases and the French paraphrase $\mathrm{f} 0$ ). This suggests that adding the source paraphrases is more effective than adding the target paraphrases.

Adding paraphrases from both sides is better than adding paraphrases from either side: The curve of adding paraphrases from both the source and the target sides is higher than both the curve of adding the target paraphrases and the curve of adding the source paraphrases. Training on 11 paraphrases from both sides, i.e., a total of 22 paraphrases achieves a BLEU score of 50.8, which is +3.8 higher than that of training on the target side only and +1.9 higher than that of training on the source side only. The advantage of combining both sides is that we can combine paraphrases from both the source and the target to reach 24 paraphrases in total to achieve a BLEU score of 57.2.

Adding both paraphrases and language families yields mixed performance: We conduct one more experiment combining the source and target paraphrases together with additional multilingual data. This is the only experiment on paraphrases where we use multilingual data other than only French and English data. The BLEU score is 49.3, higher than training on families alone, in fact, it is higher than training on eight European fami- 
lies altogether. However, it is lower than training on English and French paraphrases alone. Indeed, adding paraphrases as foreign languages is effective, however, when there is a lack of data, mixing the paraphrases with multilingual data is helpful.

Adding paraphrases increases entropy and diversity in lexical choice, and improves the sparsity issue of rare words: We use bootstrap resampling and construct $95 \%$ confidence intervals for entropies (Shannon, 1951) of all models of Vmix, i.e., models adding paraphrases at both the source and the target sides. We find that the more paraphrases, the higher the entropy, the more diversity in lexical choice as shown in Table 4. From the word F-measure shown in Table 5, we find that the more paraphrases, the better the model handles the sparsity of rare words issue. Adding paraphrases not only achieves much higher BLEU score than the WMT baseline, but also handles the sparsity issue much better than the WMT baseline.

Adding paraphrases helps rhetoric translation and increases expressiveness: Qualitative evaluation shows many cases where rhetoric translation is improved by training on diverse sets of paraphrases. In Table 3, Paraphrases help NMT to use a more contemporary synonym of "silver", "money", which is more direct and easier to understand. Paraphrases simplifies the rhetorical or subtle expressions, for example, our model uses "rejoice" to replace "break out into song", a personification device of mountains to describe joy, which captures the essence of the meaning being conveyed. However, we also observe that NMT wrongly translates "clap the palm" to "strike". We find the quality of rhetorical translation ties closely with the diversity of parallel paraphrases data. Indeed, the use of paraphrases to improve rhetoric translation is a good future research question. Please refer to the Table 3 for more qualitative examples.

\section{Conclusion}

We train on paraphrases as foreign languages in the style of multilingual NMT. Adding paraphrases improves translation quality, the rare word issue, and diversity in lexical choice. Adding the source paraphrases helps more than adding the target ones, while combining both boosts performance further. Adding multilingual data to paraphrases yields mixed performance. We would like to explore the common structure and terminology consistency across different paraphrases. Since structure and terminology are shared across paraphrases, we are interested in a building an explicit representation of the paraphrases and extend our work for better translation, or translation with more explicit and more explainable hidden states, which is very important in all neural systems.

We are interested in broadening our dataset in our future experiments. We hope to use other parallel paraphrasing corpora like the poetry dataset as shown in Table 7. There are very few poems that are translated multiple times into the same language, we therefore need to train on extremely small dataset. Rhetoric in paraphrasing is important in poetry dataset, which again depends on the training paraphrases. The limited data issue is also relevant to the low-resource setting.

We would like to effectively train on extremely small low-resource paraphrasing data. As discussed above about the potential research poetry dataset, dataset with multiple paraphrases is typically small and yet valuable. If we can train using extremely small amount of data, especially in the low-resource scenario, we would exploit the power of multi-paraphrase NMT further.

Cultural-aware paraphrasing and subtle expressions are vital (Levin et al., 1998; Larson, 1984). Rhetoric in paraphrasing is a very important too. In Figure 1, "is your sake warm enough?" in Asian culture is an implicit way of saying "would you like me to warm the sake for you?". We would like to model the culture-specific subtlety through multi-paraphrase training.

\section{References}

Rami Al-Rfou, Bryan Perozzi, and Steven Skiena. 2013. Polyglot: Distributed word representations for multilingual nlp. In Proceedings of the 17th Conference on Computational Natural Language Learning, pages 183-192, Sofia, Bulgaria. Association for Computational Linguistics.

Regina Barzilay and Kathleen R McKeown. 2001. Extracting paraphrases from a parallel corpus. In Proceedings of the 39th Annual Meeting on Association for Computational Linguistics, pages 50-57. Association for Computational Linguistics.

Florin Brad and Traian Rebedea. 2017. Neural paraphrase generation using transfer learning. In Proceedings of the 10th International Conference on Natural Language Generation, pages 257-261.

Chris Callison-Burch, Colin Bannard, and Josh Schroeder. 2005. Scaling phrase-based statistical machine translation to larger corpora and longer 
phrases. In Proceedings of the 43rd Annual Meeting on Association for Computational Linguistics, pages 255-262. Association for Computational Linguistics.

Chris Callison-Burch, Philipp Koehn, and Miles Osborne. 2006. Improved statistical machine translation using paraphrases. In Proceedings of North American Chapter of the Association for Computational Linguistics on Human Language Technologies, pages 17-24. Association for Computational Linguistics.

Daxiang Dong, Hua Wu, Wei He, Dianhai Yu, and Haifeng Wang. 2015. Multi-task learning for multiple language translation. In Proceedings of the 53rd Annual Meeting of the Association for Computational Linguistics, pages 1723-1732.

Li Dong, Jonathan Mallinson, Siva Reddy, and Mirella Lapata. 2017. Learning to paraphrase for question answering. In Proceedings of the 22nd Conference on Empirical Methods in Natural Language Processing, pages 875-886.

Marzieh Fadaee, Arianna Bisazza, and Christof Monz. 2017. Data augmentation for lowresource neural machine translation. arXiv preprint arXiv:1705.00440.

Anthony Fader, Luke Zettlemoyer, and Oren Etzioni. 2013. Paraphrase-driven learning for open question answering. In Proceedings of the 51st Annual Meeting of the Association for Computational Linguistics, pages 1608-1618.

Orhan Firat, Kyunghyun Cho, and Yoshua Bengio. 2016. Multi-way, multilingual neural machine translation with a shared attention mechanism. In Proceedings of the 15th Conference of the North American Chapter of the Association for Computational Linguistics on Human Language Technologies, pages 866-875.

Juri Ganitkevitch, Benjamin Van Durme, and Chris Callison-Burch. 2013. Ppdb: The paraphrase database. In Proceedings of the 12th Conference of the North American Chapter of the Association for Computational Linguistics on Human Language Technologies, pages 758-764.

Dan Gillick, Cliff Brunk, Oriol Vinyals, and Amarnag Subramanya. 2016. Multilingual language processing from bytes. In Proceedings of the 15th Conference of the North American Chapter of the Association for Computational Linguistics on Human Language Technologies, pages 1296-1306.

Jiatao Gu, James Bradbury, Caiming Xiong, Victor OK Li, and Richard Socher. 2017. Nonautoregressive neural machine translation. arXiv preprint arXiv:1711.02281.

Thanh-Le Ha, Jan Niehues, and Alexander Waibel. 2016. Toward multilingual neural machine translation with universal encoder and decoder. arXiv preprint arXiv:1611.04798.
Sadid A Hasan, Bo Liu, Joey Liu, Ashequl Qadir, Kathy Lee, Vivek Datla, Aaditya Prakash, and Oladimeji Farri. 2016. Neural clinical paraphrase generation with attention. In Proceedings of the Clinical Natural Language Processing Workshop, pages $42-53$.

Melvin Johnson, Mike Schuster, Quoc V Le, Maxim Krikun, Yonghui Wu, Zhifeng Chen, Nikhil Thorat, Fernanda Viégas, Martin Wattenberg, Greg Corrado, et al. 2017. Google's multilingual neural machine translation system: Enabling zero-shot translation. Transactions of the Association for Computational Linguistics, 5:339-351.

Guillaume Klein, Yoon Kim, Yuntian Deng, Jean Senellart, and Alexander Rush. 2017. Opennmt: Open-source toolkit for neural machine translation. Proceedings of the 55th annual meeting of the Association for Computational Linguistics, System Demonstrations, pages 67-72.

Mildred L Larson. 1984. Meaning-based translation: A guide to cross-language equivalence. University press of America Lanham.

Lori Levin, Donna Gates, Alon Lavie, and Alex Waibel. 1998. An interlingua based on domain actions for machine translation of task-oriented dialogues. In Proceedings of the 5th International Conference on Spoken Language Processing.

Nitin Madnani, Joel Tetreault, and Martin Chodorow. 2012. Re-examining machine translation metrics for paraphrase identification. In Proceedings of the 11th Conference of the North American Chapter of the Association for Computational Linguistics on $\mathrm{Hu}$ man Language Technologies, pages 182-190. Association for Computational Linguistics.

Jonathan Mallinson, Rico Sennrich, and Mirella Lapata. 2017. Paraphrasing revisited with neural machine translation. In Proceedings of the 15th Conference of the European Chapter of the Association for Computational Linguistics, pages 881-893.

Thomas Mayer and Michael Cysouw. 2014. Creating a massively parallel bible corpus. Oceania, 135(273):40.

Shashi Narayan, Claire Gardent, Shay Cohen, and Anastasia Shimorina. 2017. Split and rephrase. In Proceedings of the 22nd Conference on Empirical Methods in Natural Language Processing, pages 617-627.

Bo Pang, Kevin Knight, and Daniel Marcu. 2003. Syntax-based alignment of multiple translations: Extracting paraphrases and generating new sentences. In Proceedings of North American Chapter of the Association for Computational Linguistics on Human Language Technologies.

Kishore Papineni, Salim Roukos, Todd Ward, and WeiJing Zhu. 2002. Bleu: a method for automatic evaluation of machine translation. In Proceedings of 
the 40th annual meeting on association for computational linguistics, pages 311-318. Association for Computational Linguistics.

Chris Quirk, Chris Brockett, and William Dolan. 2004. Monolingual machine translation for paraphrase generation. In Proceedings of the 9th Conference on Empirical Methods in Natural Language Processing.

Yuuki Sekizawa, Tomoyuki Kajiwara, and Mamoru Komachi. 2017. Improving japanese-to-english neural machine translation by paraphrasing the target language. In Proceedings of the 4th Workshop on Asian Translation, pages 64-69.

Claude E Shannon. 1951. Prediction and entropy of printed english. Bell Labs Technical Journal, 30(1):50-64.

Yui Suzuki, Tomoyuki Kajiwara, and Mamoru Komachi. 2017. Building a non-trivial paraphrase corpus using multiple machine translation systems. In Proceedings of ACL 2017, Student Research Workshop, pages 36-42.

Yulia Tsvetkov, Sunayana Sitaram, Manaal Faruqui, Guillaume Lample, Patrick Littell, David Mortensen, Alan W Black, Lori Levin, and Chris Dyer. 2016. Polyglot neural language models: A case study in cross-lingual phonetic representation learning. In Proceedings of the 15th Conference of the North American Chapter of the Association for Computational Linguistics on Human Language Technologies, pages 1357-1366.

Liang Zhou, Chin-Yew Lin, and Eduard Hovy. 2006. Re-evaluating machine translation results with paraphrase support. In Proceedings of the 11th Conference on Empirical Methods in Natural Language Processing, pages 77-84. Association for Computational Linguistics.

Zhong Zhou, Matthias Sperber, and Alex Waibel. 2018. Massively parallel cross-lingual learning in low-resource target language translation. 2018 Third Conference on Machine Translation (WMT18), pages 232--243.

Barret Zoph and Kevin Knight. 2016. Multi-source neural translation. In Proceedings of the 15th Conference of the North American Chapter of the Association for Computational Linguistics on Human Language Technologies, pages 30-34. 
Appendix A Supplemental Materials

We show a few examples of parallel paraphrasing data in the Bible corpus. We also show some paraphrasing examples from the modern poetry dataset, which we are considering for future research. 


\begin{tabular}{|c|c|}
\hline \multirow{3}{*}{ English Paraphrases } & $\begin{array}{l}\text { Consider the lilies, how they grow: they neither toil nor spin, yet I tell you, even Solomon in } \\
\text { all his glory was not arrayed like one of these. English Standard Version. }\end{array}$ \\
\hline & $\begin{array}{l}\text { Look how the wild flowers grow! They don't work hard to make their clothes. But I tell you } \\
\text { Solomon with all his wealth wasn't as well clothed as one of these flowers. Contemporary } \\
\text { English Version. }\end{array}$ \\
\hline & $\begin{array}{l}\text { Consider how the wild flowers grow. They do not labor or spin. Yet I tell you, not even } \\
\text { Solomon in all his splendor was dressed like one of these. New International Version. }\end{array}$ \\
\hline \multirow[t]{3}{*}{ French Paraphrases } & $\begin{array}{l}\text { Considérez les lis! Ils poussent sans se fatiguer à tisser des vêtements. Et pourtant, je vous } \\
\text { l'assure, le roi Salomon lui-même, dans toute sa gloire, n'a jamais été aussi bien vêtu que l'un } \\
\text { d'eux! La Bible du Semeur. }\end{array}$ \\
\hline & $\begin{array}{l}\text { Considérez comment croissent les lis: ils ne travaillent ni ne filent; cependant je vous dis que } \\
\text { Salomon même, dans toute sa gloire, n'a pas été vêtu comme l'un d'eux. Louis Segond. }\end{array}$ \\
\hline & $\begin{array}{l}\text { Observez comment poussent les plus belles fleurs: elles ne travaillent pas et ne tissent pas; } \\
\text { cependant je vous dis que Salomon lui-même, dans toute sa gloire, n'a pas eu d'aussi belles } \\
\text { tenues que l'une d'elles. Segond } 21 \text {. }\end{array}$ \\
\hline \multirow[t]{3}{*}{ Tagalog Paraphrases } & $\begin{array}{l}\text { Wariin ninyo ang mga lirio, kung paano silang nagsisilaki: hindi nangagpapagal, o nangag- } \\
\text { susulid man; gayon ma'y sinasabi ko sa inyo, Kahit si Salomon man, sa buong kaluwalhatian } \\
\text { niya, ay hindi nakapaggayak na gaya ng isa sa mga ito. Ang Biblia } 1978 \text {. }\end{array}$ \\
\hline & $\begin{array}{l}\text { Isipin ninyo ang mga liryo kung papaano sila lumalaki. Hindi sila nagpapagal o nag-iikid. } \\
\text { Gayunman, sinasabi ko sa inyo: Maging si Solomon, sa kaniyang buong kaluwalhatian ay } \\
\text { hindi nadamitan ng tulad sa isa sa mga ito. Ang Salita ng Diyos. }\end{array}$ \\
\hline & $\begin{array}{l}\text { Tingnan ninyo ang mga bulaklak sa parang kung paano sila lumalago. Hindi sila nagtatrabaho } \\
\text { ni humahabi man. Ngunit sinasabi ko sa inyo, kahit si Solomon sa kanyang karangyaan ay } \\
\text { hindi nakapagdamit ng singganda ng isa sa mga bulaklak na ito. Magandang Balita Biblia. }\end{array}$ \\
\hline \multirow{3}{*}{ Spanish Paraphrases } & $\begin{array}{l}\text { Considerad los lirios, cómo crecen; no trabajan ni hilan; pero os digo que ni Salomón en toda } \\
\text { su gloria se vistió como uno de éstos. La Biblia de las Américas. }\end{array}$ \\
\hline & $\begin{array}{l}\text { Fíjense cómo crecen los lirios. No trabajan ni hilan; sin embargo, les digo que ni siquiera } \\
\text { Salomón, con todo su esplendor, se vestía como uno de ellos. Nueva Biblia al Día. }\end{array}$ \\
\hline & $\begin{array}{l}\text { Aprendan de las flores del campo: no trabajan para hacerse sus vestidos y, sin embargo, } \\
\text { les aseguro que ni el rey Salomón, con todas sus riquezas, se vistió tan bien como ellas. } \\
\text { Traducción en lenguaje actual. }\end{array}$ \\
\hline
\end{tabular}

Table 6: Examples of parallel paraphrasing data with English, French, Tagalog and Spanish paraphrases in Bible translation.

\begin{tabular}{|c|c|}
\hline English Original & $\begin{array}{l}\text { If you can fill the unforgiving minute with sixty seconds' worth of distance run, yours is } \\
\text { the Earth and everything that's in it, and-which is more-you'll be a Man, my son! "if", } \\
\text { Rudyard Kipling. }\end{array}$ \\
\hline \multirow[t]{3}{*}{ German Translations } & $\begin{array}{l}\text { Wenn du in unverzeihlicher Minute Sechzig Minuten lang verzeihen kannst: Dein ist die } \\
\text { Welt-und alles was darin ist- Und was noch mehr ist-dann bist du ein Mensch! Transla- } \\
\text { tion by Anja Hauptmann. }\end{array}$ \\
\hline & $\begin{array}{l}\text { Wenn du erfüllst die herzlose Minute Mit tiefstem Sinn, empfange deinen Lohn: Dein ist die } \\
\text { Welt mit jedem Attribute, Und mehr noch: dann bist du ein Mensch, mein Sohn! Translation } \\
\text { by Izzy Cartwell. }\end{array}$ \\
\hline & $\begin{array}{l}\text { Füllst jede unerbittliche Minute Mit sechzig sinnvollen Sekunden an; Dein ist die Erde dann } \\
\text { mit allem Gute, Und was noch mehr, mein Sohn: Du bist ein Mann! Translation by Lothar } \\
\text { Sauer. }\end{array}$ \\
\hline \multirow{3}{*}{ Chinese Translations } & $\begin{array}{l}\text { 若胸有激雷, 而能面如平湖, 则山川丘珡, 天地万物皆与尔共, 吾儿终成人也! Transla- } \\
\text { tion by Anonymous. }\end{array}$ \\
\hline & $\begin{array}{l}\text { 如果你能惜时如金利用每一分钟不可追回的光阴; 那公, 你的修为就会如天地般博 } \\
\text { 大, 并拥有了属于自己的世界, 更重要的是：孩子，你成为了真正顶天立地之人! } \\
\text { Translation by Anonymous. }\end{array}$ \\
\hline & $\begin{array}{l}\text { 假如你能把每一分宝贵的光阴, 化作六十秒的奋斗一你就拥有了整个世界, 最重要的 } \\
\text { 是一你就成了一个真正的人, 我的孩子! Translation by Shan Li. }\end{array}$ \\
\hline \multirow[t]{3}{*}{ Portuguese Translations } & $\begin{array}{l}\text { Se você puder preencher o valor do inclemente minuto perdido com os sessenta segundos } \\
\text { ganhos numa longa corrida, sua será a Terra, junto com tudo que nela existe, e-mais impor- } \\
\text { tante- - você será um Homem, meu filho! Translation by Dascomb Barddal. }\end{array}$ \\
\hline & $\begin{array}{l}\text { Pairando numa esfera acima deste plano, Sem receares jamais que os erros te retomem, } \\
\text { Quando já nada houver em ti que seja humano, Alegra-te, meu filho, então serás um homem!... } \\
\text { Translation by Féliz Bermudes. }\end{array}$ \\
\hline & $\begin{array}{l}\text { Se és capaz de dar, segundo por segundo, ao minuto fatal todo valor e brilho. Tua é a Terra } \\
\text { com tudo o que existe no mundo, e-o que ainda é muito mais-és um Homem, meu filho! } \\
\text { Translation by Guilherme de Almeida. }\end{array}$ \\
\hline
\end{tabular}

Table 7: Examples of parallel paraphrasing data with German, Chinese, and Portuguese paraphrases of the English poem "If by Rudyard Kipling. 